\title{
The combined effect of Parathyroid hormone (1-34) and whole-body Vibration exercise in the treatment of postmenopausal OSteoporosis (PaVOS study): a randomized controlled trial
}

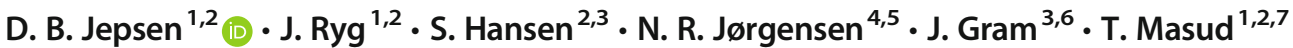

Received: 11 February 2019 / Accepted: 21 May 2019 /Published online: 15 July 2019

(C) The Author(s) 2019

\begin{abstract}
Summary Treatment effects of combining teriparatide and whole-body vibration exercise (WBV) vs teriparatide alone in twelve months were compared using bone mineral density (BMD), bone microarchitecture, and bone turnover markers. We found an increased effect in lumbar spine BMD by adding WBV to teriparatide in postmenopausal osteoporotic women.

Introduction The parathyroid hormone (PTH) analogue teriparatide is an effective but expensive anabolic treatment for osteoporosis. Whole-body vibration exercise (WBV) has been found to stimulate muscle and bone strength in some studies. Animal data demonstrate a beneficial effect on bone when combining PTH with mechanical loading. The aim of this study was to investigate if combining WBV exercise and teriparatide treatment gives additional beneficial effects on bone compared to teriparatide alone in postmenopausal women with osteoporosis.

Methods The PaVOS study is a randomized controlled trial where postmenopausal osteoporotic women starting teriparatide $20 \mu \mathrm{g} /$ day were randomized to WBV + teriparatide or teriparatide alone. WBV consisted of three sessions a week (12 min, including 1:1 ratio of exercise:rest). Bone mineral density (BMD) and bone microarchitecture, bone turnover markers, and sclerostin measurements were obtained. Data were analyzed using a linear mixed regression model with adjustment for baseline values or robust cluster regression in an intention-to-treat (ITT) analysis.

Results Thirty-five women were randomized (17 in teriparatide + WBV group and 18 in teriparatide group). At 12 months, both groups increased significantly in BMD at the lumbar spine. The teriparatide + WBV group increased by (mean \pm SD) $8.90 \% \pm$ 5.47 and the teriparatide group by $6.65 \% \pm 5.51$. The adjusted treatment effect of adding WBV to teriparatide was statistically significant at $2.95 \%[95 \% \mathrm{CI}=0.14-5.77 ; P=0.040]$. Markers of bone turnover increased significantly in both groups at three
\end{abstract}

Electronic supplementary material The online version of this article (https://doi.org/10.1007/s00198-019-05029-z) contains supplementary material, which is available to authorized users.

D. B. Jepsen

Ditte.Beck.Jepsen@rsyd.dk

J. Ryg

Jesper.Ryg@ rsyd.dk

S. Hansen

Stinus.Gadegaard.Hansen@ rsyd.dk

N. R. Jørgensen

Niklas.Rye.Joergensen@ regionh.dk

J. Gram

Jeppe.Gram@ @rsyd.dk

T. Masud

Tahir.Masud@nuh.nhs.uk
1 Department of Geriatric Medicine, Odense University Hospital, Odense, Denmark

2 Institute of Clinical Research, University of Southern Denmark, Odense, Denmark

3 Department of Endocrinology, Odense University Hospital, Odense, Denmark

4 Department of Clinical Biochemistry, Rigshospitalet, Copenhagen, Denmark

5 OPEN-Odense Patient data Explorative Network, Department of Clinical Research, University of Southern Denmark, Odense, Denmark

6 Department of Endocrinology, Hospital of Southwest Jutland, Esbjerg, Denmark

7 Department of Geriatric Medicine, Nottingham University Hospitals Trust NHS, Nottingham, UK 
and six months with no significant difference between groups. No other treatment effects were observed in hip BMD, bone microarchitecture parameters, or sclerostin levels in either group.

Conclusion Twelve months of WBV and teriparatide had a significant clinically relevant treatment effect in lumbar spine BMD compared to teriparatide alone in postmenopausal osteoporotic women.

ClinicalTrials.gov:(NCT02563353).

Keywords postmenopausal osteoporosis $\cdot$ randomized controlled trial $\cdot$ teriparatide $\cdot$ whole-body vibration

\section{Introduction}

Osteoporosis is characterized by a loss of bone mass and strength that leads to an increased risk of fragility fractures [1]. The latter are associated with substantial morbidity and increased mortality causing great negative impact on the individual but also to society in terms of cost of fracture treatment and health care $[2,3]$. In Europe, the direct medical cost of these fractures has been estimated at 31.7 billion Euros per year [4]. In an aging population, the prevalence of osteoporosis is increasing and the cost is expected to rise to 76.7 billion Euros by 2050 [4], and methods for fracture prevention are thus highly desirable.

The World Health Organization (WHO) criteria for diagnosing osteoporosis is based on measurement of areal bone mineral density (BMD), but there are also other important determinants of bone strength, including bone microarchitecture and bone turnover [1].

Whole-body vibration (WBV) has been introduced as an exercise form giving loading to bones and adjacent muscles resulting in improved muscle strength and balance $[5,6]$ and decrease in fall rates in humans [7]. Animal studies have shown that vibration therapy has anabolic effects on bone $[8$, 9], while in humans contradictory results have been found. Some studies show a positive effect on BMD at the hip [10, $11]$ or lumbar spine $[12,13]$ while others have shown no effects [14-17]. In terms of bone microarchitecture and bone turnover markers, few data are available with no overall effect shown on bone microarchitecture in the peripheral skeleton $[16,17]$. Low-magnitude WBV has shown no effect on bone turnover markers [14], while high-magnitude WBV has been shown to increase the level of the bone formation marker procollagen type $1 \mathrm{~N}$-terminal propeptide (P1NP) [18].

The parathyroid hormone (PTH) 1-34 fragment teriparatide is an effective anabolic agent for the treatment of osteoporosis. Teriparatide has been shown to increase BMD at the lumbar spine by 9.7 and $2.6 \%$ at the total hip after eighteen months of treatment and to significantly reduce the incidence of vertebral and non-vertebral fractures [19]. The bone turnover marker P1NP increases early in the treatment with teriparatide, and greater changes are associated with larger BMD increases at the lumbar spine [20, 21]. However, teriparatide is restricted to a treatment period of two years and has a high cost. Thus, it would be advantageous to optimize the effect of teriparatide if possible. In animal studies, the combination of PTH analogues and mechanical loading has shown promising results by improving the anabolic effects on bone [22-24]. One hypothesis is that WBV, like mechanical loading, can generate shear stress in the bone marrow and the osteocytic canalicular network that mediates the mechanical signal that leads to bone anabolism $[25,26]$. In animal studies that have combined WBV and PTH, some have found a positive effect of this combination on bone parameters [27-29] while other studies have found no additional effect compared to PTH alone [30, 31].

The aim of this randomized controlled trial was to investigate the effect of combining WBV and teriparatide on BMD, bone microarchitecture, and bone turnover markers compared to teriparatide alone in postmenopausal women with severe osteoporosis.

\section{Methods}

\section{Design and randomization}

A 12-month, superiority multi-centre randomized active comparator trial (RCT) with two parallel arms investigating the effect on bone parameters of combined WBV and teriparatide treatment compared to teriparatide treatment alone was conducted from November 2015 to November 2018. All participants were treated with teriparatide due to their severe osteoporosis. It was considered unethical to include a group with no pharmacological treatment due to the high fracture risk in the population participating in the study.

All participants received subcutaneous teriparatide treatment $(20 \mu \mathrm{g} / \mathrm{day})$ and were all advised to take supplements with calcium and vitamin D according to current Danish osteoporosis treatment guidelines. A computer-generated webbased block-randomization scheme with block size of 4-6 with no stratification and a 1:1 allocation was used to assign eligible participants to the intervention group (WBV and teriparatide) or active comparator group (teriparatide alone). Web-based randomization was conducted after inclusion, and baseline measurements were obtained. Demographic data were collected at baseline, and T-scores were calculated using 
reference values from the NHANES III database for the total hip and a Hologic reference material for lumbar spine provided by the manufacturer. Technicians performing bone measurements were blinded to group allocation, but sham WBV was not utilized.

\section{Participants}

Recruitment was performed in the following five Danish outpatient clinics: the Osteoporosis Clinics at Odense University Hospital (Odense and Svendborg), the Department of Geriatrics at Odense University Hospital, the Department of Endocrinology at Hospital South West Jutland, the Department of Endocrinology at Hospital Lillebaelt and the Department of Endocrinology and Internal Medicine, Aarhus University Hospital. The inclusion criteria were as follows: postmenopausal women $\geq 50$ years of age, with either one vertebral fracture within the last 3 years with $>25 \%$ reduction in vertebral height and $\mathrm{T}$-score $<-3$ at the lumbar spine or total hip, or at least two vertebral fractures with $>25 \%$ reduction in vertebral height, with no additional requirements for low BMD, planned to start treatment with teriparatide. Exclusion criteria were ongoing oral glucocorticoid treatment, inability to tolerate WBV for 1 min at screening or contraindications to WBV, such as the presence of pacemakers or joint replacements. All participants gave informed consent, and the study was approved by the Ethical Scientific Committee of Southern Denmark.

\section{Intervention and adherence}

The WBV was conducted using Power Plate My5 (Power Plate ${ }^{\circledR}$, UK), with a frequency of $30 \mathrm{~Hz}$ and amplitude of $1 \mathrm{~mm}$ (low displacement) and peak acceleration of $35.53 \mathrm{~m} \mathrm{~s}^{-2}$ root-mean-square $(3.6 \mathrm{~g})$. The training was conducted at the participants' own homes. The protocol and monitoring by attendance log has previously been described in detail in a protocol paper [32]. The WBV training protocol consisted of twelve minutes training with the WBV:rest ratio 1:1 min, including six minutes of vibration, three days a week with one day pause in between. The WBV protocol has previously been shown to be safe, feasible and anabolic to bone in an older population [18]. The WBV intervention was conducted with the knees slightly bent (at approximately $20^{\circ}$ ) to prevent vibrations causing side effects such as dizziness from the vibration transmitted to the head. Others have shown that 9-28\% of the vibration transmit to the spine measure at L3 with flexed knees using high-magnitude WBV [33]. The training gradually progressed up to the six minutes during the first eight weeks to ensure that participants could tolerate the training. We measured adherence by calculating the percentage of total conducted vibration sessions in relation to the total sessions in the training protocol, with high adherence being defined as completing $\geq 75 \%$ of the WBV sessions. Falls were assessed with calendars, and monthly telephone contact was used to ensure adherence and to collect data on pain, dizziness, falls and adverse events.

Demographic data was obtained at baseline before randomization, and physical activity was assessed using the international physical activity questionnaire (IPAQ) with the participants reporting a full week's physical activity.

Adherence to the teriparatide treatment was calculated as percentage of participants collected $\geq 80 \%$ of prescriptions in the twelve months using the Danish national electronic prescription database which stores data on prescriptions handled in all pharmacies in Denmark.

\section{Dual-energy X-ray absorptiometry}

Areal BMD of lumbar spine (L1-L4) and the total hip was measured by dual-energy X-ray absorptiometry (DXA) (Hologic Discovery, Waltham, MA, USA) at baseline and after six and twelve months of follow-up. Trained technicians acquired and analyzed all DXA scans on the same scanner according to standard Hologic protocols and performed daily quality control procedures. The coefficient of variation (CV) of the BMD measurements at the lumbar spine and total hip was $1.5 \%$ at both sites at the research unit conducting the scans.

\section{HR-pQCT}

HR-pQCT (XtremeCT; Scanco Medical, Zurich, Switzerland) was used to obtain images of the non-dominant distal radius and tibia (or the dominant side in case of previous fracture at the desired site). The standard patient protocol for image acquisition was applied as described previously [34]. In brief, a $2 \mathrm{D}$ radiograph was obtained, and the scan region of interest was positioned with a 9.5 and $22.5 \mathrm{~mm}$ offset from the radius and tibia endplate, respectively, and extended $9.02 \mathrm{~mm}$ proximally. Each image comprised of 110 slices with an isotropic $82-\mu \mathrm{m}$ voxel size. The operator immediately viewed the most distal slice for motion artefacts (e.g., blurring or cortical discontinuities), and up to two repeat acquisitions at each site were performed in case of visible motion artefacts. After reconstruction, image quality was graded by one author (DBJ) using a five-step scale as suggested by the manufacturer $(1=$ best, $5=$ worst) and images with grade $>3$ were disregarded [35]. Cortical and trabecular bone evaluation was performed using the manufacturer's default method, where trabecular bone volume per tissue volume (BV/TV) was calculated from trabecular volumetric BMD (vBMD) assuming a mineral density of fully mineralized bone of $1.2 \mathrm{mg}$ hydroxyapatite $/ \mathrm{mg}^{3}$ [36]. Trabecular number $(\mathrm{Tb} \cdot \mathrm{N})$ was extracted using a distance transformation method, whereas trabecular thickness $(\mathrm{Tb} \cdot \mathrm{Th})$ was derived from $\mathrm{BV} / \mathrm{TV}$ and $\mathrm{Tb} \cdot \mathrm{N}$. In our unit, the 
Fig. 1 Diagram showing number of subjects screened for study participation and number of participants in each treatment group at baseline and three, six, and twelve months of follow-up, with designation of reasons for early discontinuation. The intervention group receives whole-body vibration and teriparatide, and the active comparator group receives teriparatide alone

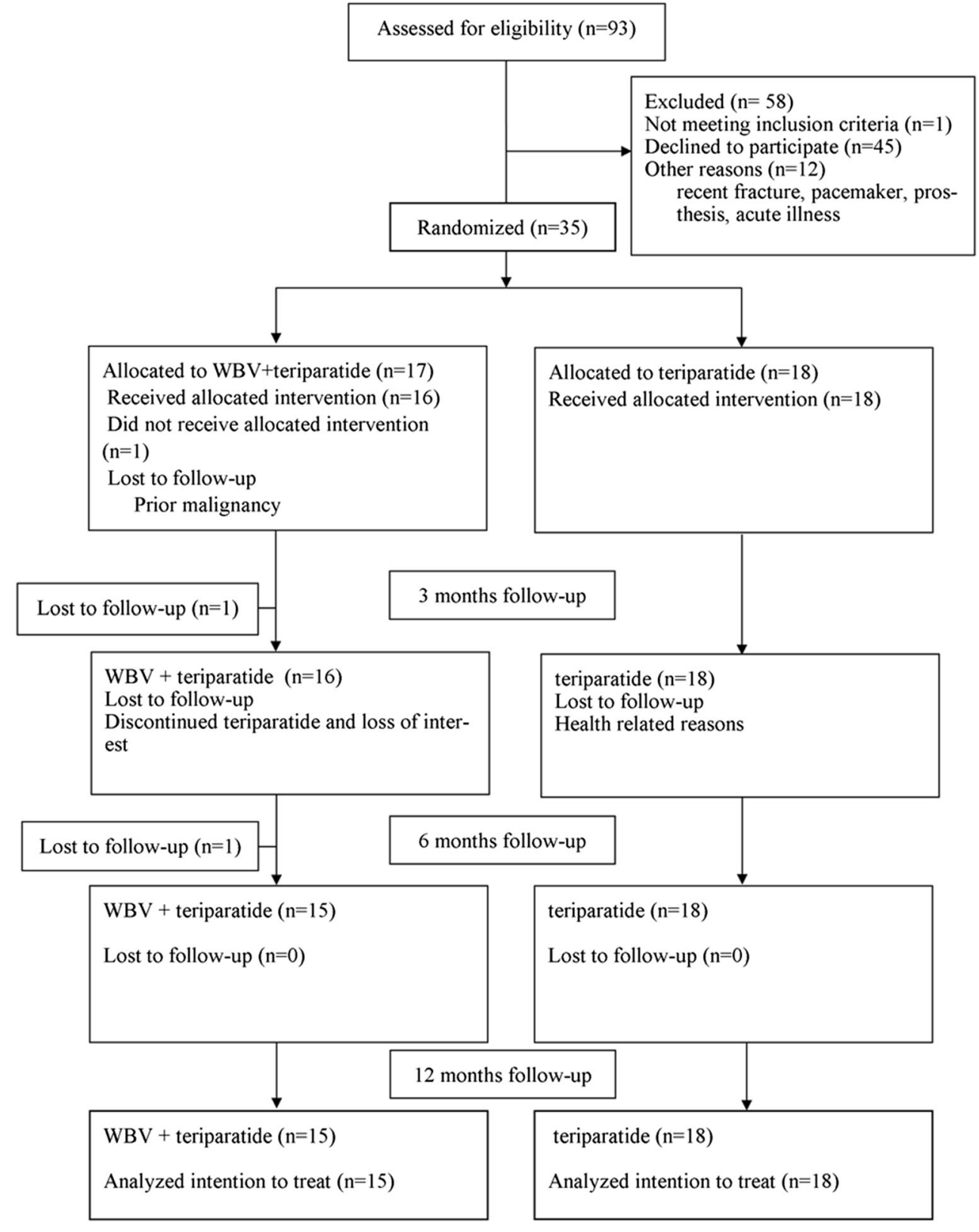

CV for geometry, vBMD, and microarchitecture measures at the radius and tibia range from 0.3 to $7.0 \%$.

\section{Bone turnover markers}

Morning blood samples were collected in EDTA plasma tubes at baseline and three and six months under standardized conditions after a minimum five minutes rest from an antecubital vein after an overnight fast. The blood was rapidly centrifuged for ten minutes, and plasma was collected and frozen. For each assay, the sample aliquots were kept frozen at $-70{ }^{\circ} \mathrm{C}$ until the day of analysis. All samples were analyzed using one single batch of each assay. Assay performance was verified using the manufacturers' control specimens. Subsequently, the plasma was analyzed for carboxy-terminal type 1 collagen crosslinks (CTX) and P1NP using Chemiluminescence (iSYS, Immunodiagnostic Systems Ltd., Boldon, England) and sclerostin using the TECOmedical Human Sclerostin HS ELISA assay (TECOmedical group, Sissach, Switzerland) at the scientific laboratory at the Department of Clinical Biochemistry, Rigshospitalet, Glostrup, Denmark. The CV for CTX, P1NP, and sclerostin ranged from 4.58 to $8.76 \%, 2.40$ to $5.69 \%$ and 6.28 to $6.52 \%$, respectively.

\section{Statistical methods}

The sample size was calculated from expected changes in the primary outcome lumbar spine BMD. The inclusion of 32 
Table 1 Baseline anthropometrics, biochemistry and DXA BMD in study participants treated with whole-body vibration (WBV) and teriparatide or teriparatide alone

\begin{tabular}{|c|c|c|c|}
\hline & $\begin{array}{l}\text { WBV + teriparatide } \\
(n=17)\end{array}$ & $\begin{array}{l}\text { Teriparatide } \\
(n=18)\end{array}$ & $P$ value \\
\hline Age, years $($ mean $\pm \mathrm{SD})$ & $69 \pm 5$ & $69 \pm 8$ & 0.909 \\
\hline Years since menopause (mean $\pm \mathrm{SD}$ ) & $22 \pm 7$ & $22 \pm 5$ & 0.979 \\
\hline Lumbar spine $\mathrm{BMD}, \mathrm{g} / \mathrm{cm}^{2}($ mean $\pm \mathrm{SD})$ & $0.674 \pm 0.103$ & $0.729 \pm 0.137$ & 0.183 \\
\hline Total hip BMD, $\mathrm{g} / \mathrm{cm}^{2}($ mean $\pm \mathrm{SD})$ & $0.618 \pm 0.096$ & $0.615 \pm 0.106$ & 0.931 \\
\hline T-score total hip (mean \pm SD) & $-2.7 \pm 0.8$ & $-2.8 \pm 0.9$ & 0.931 \\
\hline T-score lumbar spine (mean $\pm \mathrm{SD})$ & $-3.4 \pm 0.9$ & $-2.9 \pm 1.2$ & 0.183 \\
\hline Previous bisphosphonate usea, $n(\%)$ & $5(29)$ & $10(55)$ & 0.118 \\
\hline Vitamin $\mathrm{D}$ before treatment $\mathrm{t}^{\mathrm{a}}, \mathrm{nmol} / \mathrm{L}($ mean $\pm \mathrm{SD})$ & $83 \pm 24$ & $84 \pm 27$ & 0.940 \\
\hline Vitamin D, $\mu \mathrm{g} /$ day $\not$, median (IQR) & $29(17-49.5)$ & $30(19-40)$ & 0.758 \\
\hline Daily calcium supplement, mg, median (IQR) & $800(800-800)$ & $800(400-800)$ & 0.303 \\
\hline \multicolumn{4}{|l|}{ Physical activity at baseline, $n$} \\
\hline$(\%)$ & & & 0.421 \\
\hline Low & $10(59)$ & $8(39)$ & \\
\hline Moderate & $4(23)$ & $8(50)$ & \\
\hline High* & $3(18)$ & $2(11)$ & \\
\hline
\end{tabular}

$P$ values from T-tests when normally distributed, chi-squared for categorical variables, Wilcoxon rank-sum test for continuous non-normally distributed,

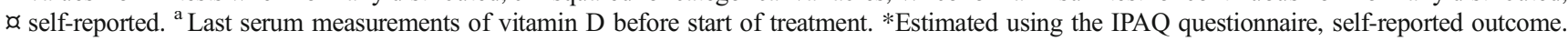
$\mathrm{BMD}$, bone mineral density; BMI, body mass index

participants (16 in each group) would give the study $80 \%$ power to detect an additional increase of $22 \%$ in BMD with WBV assuming a $9 \%$ increase of BMD in the active comparator group and $11 \%$ increase in the intervention group, and assuming a SD of the BMD increase of $2 \%$ with a level of significance of 0.05 [10]. Allowing for $20 \%$ drop out rate, the inclusion of 40 participants was planned.

All statistical analyses were performed using the Statistical Package STATA (version 14 and 15, Stata Corp., College Station, TX, USA). Between-group differences in baseline characteristics and study adverse events were assessed using the Student's T-test, Wilcoxon rank-sum or the chi-squared test according to continues outcomes, normal or non-normal distributions or categorical outcomes, respectively. Because data represent repeated measures in individuals, we evaluated the differences in BMD over time as percent change from baseline between those treated with $\mathrm{WBV}+$ teriparatide and teriparatide using pre-planned multi-level mixed-effects linear regression models adjusted for baseline BMD (ANCOVA) [32]. Models included a group variable (WBV + teriparatide or teriparatide) and a time variable to assess if the overall level of the parameters differed between groups and whether the parameters changed over time. Longitudinal absolute changes in microarchitecture were compared using a linear mixedeffect model with fixed effect for treatment group, time and a time and treatment interaction term, with adjustment for baseline values. Biochemical markers of bone turnover and sclerostin had a non-normal distribution and unequal variation in the residuals and were analyzed using linear regression with a robust cluster estimation in order to accommodate for possible correlations between different measurements within one individual. Model assumptions were checked using histograms, plots of the residuals against fitted values and normal probability plots of the residuals. A significance level of 0.05 was chosen. For missing data, multiple imputations were planned when appropriate depending on amount and causation [37]. We did not apply correction for multiple testing. ITT analyses was performed primarily, and a secondary per protocol (PP) analysis was conducted to investigate if WBV adherence (>75\%) affected the results. Intention-to-treat analysis (ITT) was used as pre-specified in the published study protocol [32].

\section{Results}

\section{Participants}

The recruitment centres informed 93 eligible women who were starting teriparatide treatment about potential participation in the study (Fig. 1; consort flow chart). Thirty-five postmenopausal women with a mean age $( \pm \mathrm{SD})$ of $69 \pm 7$ (range 53-81) years were recruited. Baseline characteristics of the study population are shown in Table 1. There were no significant differences in age, BMI, BMD or other demographic factors between the two groups. Of the 35 included 
Table 2 Lumbar spine and total hip BMD by DXA at baseline, 6 months and 12 months of follow-up in patients treated with whole-body vibration (WBV) and teriparatide or teriparatide alone

\begin{tabular}{|c|c|c|c|c|}
\hline & \multicolumn{2}{|c|}{$\mathrm{WBV}+$ teriparatide $(n=17)$} & \multicolumn{2}{|c|}{ Teriparatide $(n=18)$} \\
\hline & Mean $\pm \mathrm{SD}$ & $\begin{array}{l}\text { Change from baseline } \% \\
\text { Mean } \pm \text { SD } \\
(n=15)\end{array}$ & Mean \pm SD & $\begin{array}{l}\text { Change from baseline } \% \\
\text { Mean } \pm \text { SD } \\
(n=18)\end{array}$ \\
\hline \multicolumn{5}{|c|}{ Lumbar spine BMD $\left(\mathrm{g} / \mathrm{cm}^{2}\right)$} \\
\hline Baseline & $0.674 \pm 0.103$ & & $0.729 \pm 0.137$ & \\
\hline 6 months & $0.697 \pm 0.090$ & $6.47^{*} \pm 3.40$ & $0.759 \pm 0.160$ & $\mathbf{3 . 4 8} \pm 4.39$ \\
\hline 12 months & $0.713 \pm 0.098$ & $8.90 * \pm 5.48$ & $0.779 \pm 0.160$ & $\mathbf{6 . 6 5} \pm 5.57$ \\
\hline \multicolumn{5}{|c|}{ Total hip BMD $\left(\mathrm{g} / \mathrm{cm}^{2}\right)$} \\
\hline Baseline & $0.618 \pm 0.096$ & & $0.615 \pm 0.106$ & \\
\hline 6 months & $0.607 \pm 0.105$ & $-0.95 \pm 5.42$ & $0.613 \pm 0.103$ & $-0.12 \pm 3.94$ \\
\hline 12 months & $0.611 \pm 0.100$ & $-0.18 \pm 4.08$ & $0.622 \pm 0.110$ & $0.81 \pm 4.11$ \\
\hline
\end{tabular}

Parameters printed in bold indicate a significant increase within group from baseline $P<0.05$. * indicates a significant difference between groups $P<0.05$. BMD, bone mineral density. $P$ values derived from mixed-effect models for longitudinal percent change from baseline with fixed effect for treatment groups, time and adjustments for baseline measurements in an intention-to-treat analysis

participants, $32(91.4 \%)$ completed 6 months BMD measurements and $33(95 \%)$ completed 12 months BMD measurements. Due to a low number of missing observations, no imputation was applied to BMD measurements. In the intervention group, one participant never started teriparatide and was lost to follow-up after baseline measurements, another participant was lost to follow-up after three months and two participants discontinued the WBV at three months but attended the follow-up visits. Due to technical failure of the HR-pQCT scanner in two separate periods, a total of twelve measurements were missing and exchanged by multiple imputations. One participant had no baseline radius scan due to prior fractures and no imputations were applied. In the active comparator group, three participants had missing values in the bone turnover markers and one participant did not have BMD measurements at the six-month visit. Furthermore, one participant was non-fasting and, therefore, was not included in the CTX analyses, and P1NP measurements were excluded once in two participants following an acute fracture. Missing values were exchanged by multiple imputations. One set of measurements was completely missing at random, and no values for this set were imputed.

\section{Bone mineral density}

Lumbar spine BMD increased significantly in both groups at six and twelve months. Mean percent change in the WBV+ teriparatide group at six months and twelve months was $6.47 \% \pm 3.40$ and $8.90 \% \pm 5.48$ compared to $3.48 \% \pm 4.39$ and $6.65 \% \pm 5.57$ in the teriparatide group, respectively (Table 2). The predefined primary adjusted mixed-effect model found a significant overall difference in treatment effect between groups of $2.95 \%$ [95\% CI $(0.14-5.77), P=0.04$ ].

There was no significant change in total hip BMD in the two groups or when comparing treatment effects between groups at six or twelve months (Table 2). In PP analysis with adherence $>75 \%$, both groups increased significantly in lumbar spine BMD with no significant treatment effect although a positive trend was seen in favor of the intervention $(P=0.077)$ $(n=13)$. There were no changes in the results from total hip when applying the PP analysis (Supplementary material table 1).

\section{Microarchitecture parameters}

Table 3 shows the microarchitecture parameters for the two groups. There were no significant changes from baseline in total vBMD, cortical thickness, BV/TV, trabecular number or trabecular thickness in either group, and no significant differences between groups. Moreover, there were no significant changes in microarchitecture parameters when applying the PP analysis (adherence $>75 \%$ ) (Supplementary material table 2).

\section{Bone turnover markers}

CTX and P1NP increased significantly in both groups after three and six months of follow-up. There were no significant differences between groups (Table 4). Sclerostin levels showed no significant change within or between groups. There were no significant changes in results from the bone turnover markers when applying the PP analysis (adherence $>$ $75 \%$ ) (Supplementary material table 3 ). 
Table 3 Radius and tibia HR-pQCT parameters of volumetric BMD and microarchitecture at baseline, 6 months and 12 months of follow-up in patients treated with whole-body vibration (WBV) and teriparatide or teriparatide alone

\begin{tabular}{|c|c|c|c|c|c|c|}
\hline \multicolumn{4}{|l|}{$\begin{array}{l}\text { WBV + teriparatide }(n=17) \\
\text { Mean } \pm \text { SD }\end{array}$} & \multicolumn{3}{|c|}{$\begin{array}{l}\text { Teriparatide }(n=18) \\
\text { Mean } \pm \text { SD }\end{array}$} \\
\hline Radius & $\begin{array}{l}\text { Baseline } \\
(n=16)\end{array}$ & $\begin{array}{l}6 \text { months } \\
(n=12)\end{array}$ & $\begin{array}{l}12 \text { months } \\
(n=14)\end{array}$ & $\begin{array}{l}\text { Baseline } \\
(n=18)\end{array}$ & $\begin{array}{l}6 \text { months } \\
(n=15)\end{array}$ & $\begin{array}{l}12 \text { months } \\
(n=16)\end{array}$ \\
\hline Total vBMD $\left(\mathrm{g} / \mathrm{cm}^{3}\right)$ & $196 \pm 62$ & $195 \pm 80$ & $184 \pm 73$ & $204 \pm 74$ & $215 \pm 78$ & $208 \pm 85$ \\
\hline Cortical thickness (mm) & $0.43 \pm 0.17$ & $0.43 \pm 0.21$ & $0.40 \pm 0.19$ & $0.47 \pm 0.18$ & $0.50 \pm 0.20$ & $0.46 \pm 0.22$ \\
\hline $\mathrm{BV} / \mathrm{TV}(\%)$ & $7.68 \pm 2.88$ & $7.39 \pm 3.16$ & $7.14 \pm 3.03$ & $7.63 \pm 3.34$ & $7.93 \pm 3.52$ & $7.91 \pm 3.69$ \\
\hline Trabecular no. $\left(\mathrm{mm}^{-1}\right)$ & $1.38 \pm 0.37$ & $1.36 \pm 0.46$ & $1.34 \pm 0.41$ & $1.33 \pm 0.54$ & $1.34 \pm 0.51$ & $1.41 \pm 0.48$ \\
\hline Trabecular thickness (mm) & $0.056 \pm 0.012$ & $0.055 \pm 0.017$ & $0.054 \pm 0.017$ & $0.059 \pm 0.018$ & $0.060 \pm 0.018$ & $0.055 \pm 0.013$ \\
\hline Tibia & Baseline $(n=17)$ & 6 months $(n=12)$ & 12 months $(n=14)$ & Baseline $(n=18)$ & 6 months $(n=15)$ & 12 months $(n=16)$ \\
\hline Total vBMD $\left(\mathrm{g} / \mathrm{cm}^{3}\right)$ & $174 \pm 445$ & $177 \pm 53$ & $165 \pm 50$ & $175 \pm 53$ & $180 \pm 54$ & $173 \pm 52$ \\
\hline Cortical thickness (mm) & $0.53 \pm 0.23$ & $0.58 \pm 0.26$ & $0.52 \pm 0.24$ & $0.59 \pm 0.30$ & $0.63 \pm 0.31$ & $0.57 \pm 0.30$ \\
\hline $\mathrm{BV} / \mathrm{TV}(\%)$ & $9.33 \pm 2.95$ & $9.15 \pm 4.01$ & $8.66 \pm 3.36$ & $8.7 \pm 3.06$ & $8.82 \pm 3.47$ & $8.70 \pm 3.34$ \\
\hline Trabecular no. $\left(\mathrm{mm}^{-1}\right)$ & $1.42 \pm 0.50$ & $1.35 \pm 0.56$ & $1.33 \pm 0.49$ & $1.34 \pm 0.45$ & $1.32 \pm 0.49$ & $1.36 \pm 0.48$ \\
\hline Trabecular thickness (mm) & $0.068 \pm 0.016$ & $0.071 \pm 0.020$ & $0.067 \pm 0.018$ & $0.066 \pm 0.013$ & $0.068 \pm 0.015$ & $0.065 \pm 0.015$ \\
\hline
\end{tabular}

No significant differences in change within or between groups. $P$ values are derived from mixed-effect linear regression models for longitudinal change with multiple imputations for missing values. BV/TV, trabecular bone volume per tissue volume; vBMD, volumetric bone mineral density; no., number

\section{Adherence}

A total of 13 participants in the intervention group reported $>$ $75 \%$ training adherence with the WBV $(76 \%$ of the participants). Two participants reported pain in the lower extremities, and one stopped the WBV.

A total of 33 participants (94\%) had an adherence of $>80 \%$ to teriparatide based on the number of collected prescriptions (of planned thirteen a year). Due to mild hypercalcemia, a short period off teriparatide treatment was necessary in one patient. There were no significant differences between groups in adherence to teriparatide.

A few serious adverse events (AE) were reported, which are as follows: five hospital admissions (four in the active comparator and one in the intervention group), one distal femur fracture (intervention group), and one vertebral fracture (intervention group). No serious adverse events were believed to be related to WBV. In the twelve-month intervention period, two participants in the active comparator group reported falls and five participants in the intervention group, none in relation to WBV sessions. There were no significant differences in falls between the two groups. There were no significant changes in reported pain or dizziness within the groups or between groups during the twelve months.

\section{Discussion}

In this randomized controlled trial, we investigated the combined effect of twelve-month WBV and teriparatide compared to teriparatide alone in postmenopausal women with severe osteoporosis. In the lumbar spine, the intervention resulted in an $8.90 \%$ increase in BMD from baseline compared to $6.65 \%$ in the active comparator group. The addition of WBV to teriparatide showed no additional effects in the hip BMD, bone microarchitecture parameters in radius or tibia or bone turnover markers. We did not find an increased effect in the participants with high adherence $(>75 \%)$ on lumbar spine BMD, which could be due to low power related to the limited sample size.

Teriparatide has proved to be an effective anabolic treatment in patients with osteoporosis with the most pronounced effect in lumbar spine, i.e. trabecular bone with 12 months treatment yielding an effect in lumbar spine at $6.2 \%$, while BMD at the total hip increased by $0.7 \%$ in another study with women aged 45 or older [38]. Eighteen months of teriparatide increased lumbar spine by $9.7 \% \pm 7.4$ in postmenopausal women [19], whereas our study of teriparatide and WBV resulted in an $8.9 \% \pm 5.47$ increase in lumbar spine BMD after 12 months. In line with a previous study [38], no statistically significant effect in either group could be detected in the total hip BMD in our study, possibly due to a small study population or due to the possibility that although modeling had occurred there had not been enough time for mineralization to have occurred. Another potential explanation is that the combined treatment of WBV and teriparatide may not have an added effect on BMD in the hip. However, larger studies are needed in order to make any final conclusions on this.

To our knowledge, this is the first trial combining WBV with teriparatide in postmenopausal women. A recent RCT investigated the combination of WBV and teriparatide in younger patients with chronic spinal cord injury and found 
Table 4 Serum bone turnover markers at baseline, 3 months and 6 months of follow-up in patients treated with whole-body vibration (WBV) and teriparatide or teriparatide alone

\begin{tabular}{|c|c|c|c|c|c|c|}
\hline & \multicolumn{3}{|c|}{$\begin{array}{l}\text { WBV }+ \text { teriparatide }(n=17) \\
\text { Mean } \pm \text { SD }\end{array}$} & \multicolumn{3}{|c|}{$\begin{array}{l}\text { Teriparatide }(n=17) \\
\text { Mean } \pm \text { SD }\end{array}$} \\
\hline & Baseline & 3 months & 6 months & Baseline & 3 months & 6 months \\
\hline CTX (ng/L) & $519.0 \pm 269.8$ & $919.8 \pm 468.1$ & $\mathbf{1 1 3 9 . 3} \pm 857.2$ & $390.2 \pm 356.5$ & $\mathbf{9 1 2 . 4 5} \pm 1096.9$ & $\mathbf{7 5 8 . 0} \pm 547.8$ \\
\hline P1NP $(\mu \mathrm{g} / \mathrm{L})$ & $61.8 \pm 19.7$ & $\mathbf{1 5 4 . 3 8} \pm 81.2$ & $212.93 \pm 116.6$ & $52.7 \pm 29.4$ & $\mathbf{1 5 3 . 2} \pm 105.6$ & $167.3 \pm 84.9$ \\
\hline Sclerostin (ng/mL) & $0.65 \pm 0.1$ & $0.67 \pm 0.1$ & $0.68 \pm 0.2$ & $0.69 \pm 0.2$ & $0.72 \pm 0.4$ & $0.73 \pm 0.2$ \\
\hline
\end{tabular}

Parameters printed in bold indicate significant change from baseline within groups $P<0.05$. $P$ values derived from regression model with robust cluster estimation with multiple imputations for missing values. Intention-to-treat analysis. CTX, carboxy-terminal type 1 collagen crosslinks; P1NP, procollagen type $1 \mathrm{~N}$-terminal propeptide

no additional increase in BMD, bone microarchitecture or bone turnover markers when vibration therapy was added to teriparatide [39]. The participants had a BMD increase in the lumbar spine of 4.8-5.5\% [39], which is lower than our teriparatide group receiving the same dose of teriparatide at twelve months. There are some differences between the two trials that may explain the different results. The vibration protocols used differ in vibration magnitude and training position where the study on patients with chronic spinal cord injury [39] used a seated training position with low-magnitude vibration with a root-mean-square acceleration of $0.5 \mathrm{~g}$ while our trial used standing training with a root-mean-square acceleration of $2.5 \mathrm{~g}$. Animal studies have shown that disuse or unloading can diminish the effect of PTH [40], and one hypothesis is that spinal cord injury in combination with the low magnitude vibration and the seated training position had lower muscle activation and bone loading that could cause an inferior response.

We did not find an effect in any of the groups on bone microarchitecture parameters. Other studies with longer follow-up have shown a decrease in cortical bone mineral density in radius and tibia after 18 months of teriparatide treatment [41]. Another study with WBV as a single intervention showed no significant effects in bone microarchitecture outcomes with low-magnitude WBV as a single intervention [17]. A recent systematic review found no effect on areal BMD in peripheral skeletal sites after twelve months of PTH analogues treatment but a decrease in areal BMD was detected in radius with larger doses after 18 months of treatment [42]. Regarding bone turnover markers, the increase in CTX and P1NP was significant in both groups in our study with no difference between groups. Others have reported that the increase in P1NP after three months was associated with the increase in lumbar spine and hip BMD after 12 months treatment with teriparatide, but this was in a much larger study population [43]. A prior study demonstrated that teriparatide treatment of postmenopausal women resulted in a decrease in circulating serum sclerostin levels [44], while our results are in line with a recent study showing that teriparatide treatment did not reduce the sclerostin levels in postmenopausal women [45].

There are some limitations to our study. Participants selfadministered teriparatide and WBV was performed unsupervised, and we do not know if the instructions were followed, even though in order to improve adherence, we made monthly telephone contact. Adherence was assessed through training logbooks previously reported to be reliable with an overall intra-class correlation of 0.96 compared to electronic monitoring [46]. As true WBV cannot be completely masked, doubleblinding was not possible. We did not use sham platforms that produce an audible sound and which have been used in a previous study [17]. The participants were not blinded but instructed not to reveal the allocation to the measuring technicians, and, in our opinion, the endpoints were not at risk of performance bias. The study had a small population of only 13 participants following the training protocol with adherence over $75 \%$, giving a low power to detect a possible effect in bone microarchitecture from the WBV and teriparatide interaction. Finally, we had over $10 \%$ missing observations replaced by multiple imputations in the HR-pQCT measurements which are likely to have affected results in the microarchitecture evaluation. The study duration was limited to twelve months to ensure a high level of persistence and adherence to WBV and teriparatide. It is possible that the changes in bone may have been greater with longer duration of WBV or longer follow-up.

Our study also has some important strengths. We studied a homogeneous group of osteoporotic patients (postmenopausal women). The study had a low drop-out rate with $95 \%$ of the enrolled participants returning for twelve-month DXA scans. The at-home training design ensured high training availability, and the frequent contact ensured a high attendance at study visits. Last, we examined BMD, bone microarchitecture and bone turnover markers for a comprehensive assessment of the combined effect of WBV and teriparatide on bone.

To conclude, we found a significantly larger and clinically relevant overall increase in lumbar spine BMD in the WBV + 
teriparatide group compared to the teriparatide group. There was no effect on hip BMD with either intervention and no difference between the groups. The addition of WBV might provide an enhanced anabolic bone response at the lumbar spine in postmenopausal women treated with teriparatide, although we did not detect effects on indices of bone microarchitecture in the radius or tibia or on markers of bone turnover.

Acknowledgements We would like to thank all participants and the inclusion centres. We are grateful to Mrs. Steffanie Anthony Christiansen and Mrs. Jane Nielsen for the study coordination. The team would also like to thank Mrs. Pernille Herman for the advice and support during the study.

Funding The study was funded by The Odense University Hospital Research Foundation, the Region of Southern Denmark PhD Foundation, The Research Foundation between Odense University Hospital and Rigshospitalet, OPEN - Odense Patient data Explorative Network, The A.P. Møller Foundation for the Advancement of Medical Science, and the Danish Osteoporosis Foundation. The biobank and data management was conducted in collaboration with OPEN-Odense Patient data Explorative Network. The funding parties had no influence on the study design, study conduct, results or dissemination of the results.

\section{Compliance with ethical standards}

Ethics approval and consent to participate The study was conducted according to the standards of International Conference on Harmonization, Research Ethics Committee regulations, any applicable government regulations (e.g. The Danish Data Protection Agency) and local procedures. The trial was approved by the Regional Scientific Ethical Committee of Southern Denmark (ref. ID S-20150121) and The Danish Data Protection Agency (2008-58-0035, 13/40496) and registered at ClinicalTrials.gov (NCT02563353). All participants gave informed written consent prior to enrolment.

\section{Conflicts of interest None.}

Open Access This article is distributed under the terms of the Creative Commons Attribution-NonCommercial 4.0 International License (http:// creativecommons.org/licenses/by-nc/4.0/), which permits any noncommercial use, distribution, and reproduction in any medium, provided you give appropriate credit to the original author(s) and the source, provide a link to the Creative Commons license, and indicate if changes were made.

\section{References}

1. (1994) Assessment of fracture risk and its application to screening for postmenopausal osteoporosis. Report of a WHO Study Group. World Health Organ Tech Rep Ser 843:1-129

2. Borgstrom F et al (2006) Costs and quality of life associated with osteoporosis-related fractures in Sweden. Osteoporos Int 17(5): $637-650$

3. Hernlund E et al (2013) Osteoporosis in the European Union: medical management, epidemiology and economic burden. A report prepared in collaboration with the International Osteoporosis
Foundation (IOF) and the European Federation of Pharmaceutical Industry Associations (EFPIA). Arch Osteoporos 8:136

4. Kanis JA, Johnell O (2005) Requirements for DXA for the management of osteoporosis in Europe. Osteoporos Int 16(3):229-238

5. Lam FM et al (2012) The effect of whole body vibration on balance, mobility and falls in older adults: a systematic review and metaanalysis. Maturitas 72(3):206-213

6. Sitja-Rabert $\mathrm{M}$ et al (2012) Efficacy of whole body vibration exercise in older people: a systematic review. Disabil Rehabil 34(11): 883-893

7. Jepsen DB, Thomsen K, Hansen S, Jørgensen NR, Masud T, Ryg J (2017) Effect of whole-body vibration exercise in preventing falls and fractures: a systematic review and meta-analysis. BMJ Open 7(12): 018342

8. Rubin C et al (2001) Anabolism. Low mechanical signals strengthen long bones. Nature 412(6847):603-604

9. Christiansen BA, Silva MJ (2006) The effect of varying magnitudes of whole-body vibration on several skeletal sites in mice. Ann Biomed Eng 34(7):1149-1156

10. Verschueren SM, Roelants M, Delecluse C, Swinnen S, Vanderschueren D, Boonen S (2004) Effect of 6-month whole body vibration training on hip density, muscle strength, and postural control in postmenopausal women: a randomized controlled pilot study. J Bone Miner Res 19(3):352-359

11. Beck BR, Norling TL (2010) The effect of 8 mos of twice-weekly low- or higher intensity whole body vibration on risk factors for postmenopausal hip fracture. Am J Phys Med Rehabil 89(12):997_ 1009

12. de Oliveira, LC, de Oliveira, RG, de Almeida Pires-Oliveira, DA (2019). Effects of whole-body vibration versus pilates exercise on bone mineral density in postmenopausal women: a randomized and controlled clinical trial. J Geriatr Phys Ther 42(2):E23-E31

13. Lai CL, Tseng SY, Chen CN, Liao WC, Wang CH, Lee MC, Hsu PS (2013) Effect of 6 months of whole body vibration on lumbar spine bone density in postmenopausal women: a randomized controlled trial. Clin Interv Aging 8:1603-1609

14. Kiel DP, Hannan MT, Barton BA, Bouxsein ML, Sisson E, Lang T, Allaire B, Dewkett D, Carroll D, Magaziner J, Shane E, Leary ET, Zimmerman S, Rubin CT (2015) Low-magnitude mechanical stimulation to improve bone density in persons of advanced age: a randomized, placebo-controlled trial. J Bone Miner Res 30(7): $1319-1328$

15. Gomez-Cabello A et al (2014) Effects of a short-term whole body vibration intervention on bone mass and structure in elderly people. J Sci Med Sport 17(2):160-164

16. Slatkovska L, Alibhai SMH, Beyene J, Hu H, Demaras A, Cheung $\operatorname{AM}(2011)$ Effect of 12 months of whole-body vibration therapy on bone density and structure in postmenopausal women: a randomized trial. Ann Intern Med 155(10):668-679 w205

17. Liphardt AM, Schipilow J, Hanley DA, Boyd SK (2015) Bone quality in osteopenic postmenopausal women is not improved after 12 months of whole-body vibration training. Osteoporos Int 26(3): $911-920$

18. Corrie H, Brooke-Wavell K, Mansfield NJ, Cowley A, Morris R, Masud T (2015) Effects of vertical and side-alternating vibration training on fall risk factors and bone turnover in older people at risk of falls. Age Ageing 44(1):115-122

19. Neer RM et al (2001) Effect of parathyroid hormone (1-34) on fractures and bone mineral density in postmenopausal women with osteoporosis. N Engl J Med 344(19):1434-1441

20. Blumsohn A et al (2011) Early changes in biochemical markers of bone turnover and their relationship with bone mineral density changes after 24 months of treatment with teriparatide. Osteoporos Int 22(6):1935-1946

21. Bauer DC, Garnero P, Bilezikian JP, Greenspan SL, Ensrud KE, Rosen CJ, Palermo L, Black DM, for the PTH and Alendronate 
(PaTH) Research Group (2006) Short-term changes in bone turnover markers and bone mineral density response to parathyroid hormone in postmenopausal women with osteoporosis. J Clin Endocrinol Metab 91(4):1370-1375

22. Ma Y, Jee WSS, Yuan Z, Wei W, Chen H, Pun S, Liang H, Lin C (1999) Parathyroid hormone and mechanical usage have a synergistic effect in rat tibial diaphyseal cortical bone. J Bone Miner Res 14(3):439-448

23. Roberts MD, Santner TJ, Hart RT (2009) Local bone formation due to combined mechanical loading and intermittent hPTH-(1-34) treatment and its correlation to mechanical signal distributions. J Biomech 42(15):2431-2438

24. Li J, Duncan RL, Burr DB, Gattone VH, Turner CH (2003) Parathyroid hormone enhances mechanically induced bone formation, possibly involving L-type voltage-sensitive calcium channels. Endocrinology 144(4):1226-1233

25. Coughlin TR, Niebur GL (2012) Fluid shear stress in trabecular bone marrow due to low-magnitude high-frequency vibration. $\mathrm{J}$ Biomech 45(13):2222-2229

26. Turner CH, Forwood MR, Otter MW (1994) Mechanotransduction in bone: do bone cells act as sensors of fluid flow? FASEB J 8(11): 875-878

27. Takeshi Matsumoto, Daisuke Sato, Yoshihiro Hashimoto, (2015) Individual and combined effects of noise-like whole-body vibration and parathyroid hormone treatment on bone defect repair in ovariectomized mice. Proceedings of the Institution of Mechanical Engineers, Part H: Journal of Engineering in Medicine 230 (1): 30-38

28. Shibamoto A et al (2018) Effect of high-frequency loading and parathyroid hormone administration on peri-implant bone healing and osseointegration. Int J Oral Sci 10(1):6

29. Jenifer Freitas Campos, Aline Gomes Hidalgo Mierzwa, Mariana Freitas-Jesus, Marise Lazaretti-Castro, Keico Okino Nonaka, Rejane Daniele Reginato, (2018) Mechanical Vibration Associated With Intermittent PTH Improves Bone Microarchitecture in Ovariectomized Rats. Journal of Clinical Densitometry

30. Lynch MA, Brodt MD, Stephens AL, Civitelli R, Silva MJ (2011) Low-magnitude whole-body vibration does not enhance the anabolic skeletal effects of intermittent PTH in adult mice. J Orthp Res 29(4):465-472

31. Hoffmann DB, Sehmisch S, Hofmann AM, Eimer C, Komrakova M, Saul D, Wassmann M, Stürmer KM, Tezval M (2017) Comparison of parathyroid hormone and strontium ranelate in combination with whole-body vibration in a rat model of osteoporosis. $\mathrm{J}$ Bone Miner Metab 35(1):31-39

32. Jepsen DB et al (2018) The combined effect of Parathyroid hormone (1-34) and whole-body Vibration exercise in the treatment of OSteoporosis (PaVOS) — study protocol for a randomized controlled trial. Trials 19(1):186

33. Tankisheva E, Jonkers I, Boonen S, Delecluse C, Harry van Lenthe G, Druyts HLJ, Spaepen P, Verschueren SMP (2013) Transmission of whole-body vibration and its effect on muscle activation. J Strength Cond Res 27(9):2533-2541

34. Boutroy S, Bouxsein ML, Munoz F, Delmas PD (2005) In vivo assessment of trabecular bone microarchitecture by highresolution peripheral quantitative computed tomography. J Clin Endocrinol Metab 90(12):6508-6515

35. Pauchard Y, Liphardt AM, Macdonald HM, Hanley DA, Boyd SK (2012) Quality control for bone quality parameters affected by subject motion in high-resolution peripheral quantitative computed tomography. Bone 50(6):1304-1310

36. Laib A, Hauselmann HJ, Ruegsegger P (1998) In vivo high resolution 3D-QCT of the human forearm. Technol Health Care 6(5-6): 329-337

37. Jakobsen JC et al (2017) When and how should multiple imputation be used for handling missing data in randomised clinical trials - a practical guide with flowcharts. BMC Med Res Methodol 17(1): 162

38. Tsai JN, Uihlein AV, Lee H, Kumbhani R, Siwila-Sackman E, McKay EA, Burnett-Bowie SAM, Neer RM, Leder BZ (2013) Teriparatide and denosumab, alone or combined, in women with postmenopausal osteoporosis: the DATA study randomised trial. Lancet 382(9886):50-56

39. Edwards WB, Simonian N, Haider IT, Anschel AS, Chen D, Gordon KE, Gregory EK, Kim KH, Parachuri R, Troy KL, Schnitzer TJ (2018) Effects of teriparatide and vibration on bone mass and bone strength in people with bone loss and spinal cord injury: a randomized, controlled trial. J Bone Miner Res 33(10): 1729-1740

40. Turner RT, Lotinun S, Hefferan TE, Morey-Holton E (2006) Disuse in adult male rats attenuates the bone anabolic response to a therapeutic dose of parathyroid hormone. J Appl Physiol (1985) 101(3): 881-886

41. Hansen S, Hauge EM, Beck Jensen JE, Brixen K (2013) Differing effects of PTH 1-34, PTH 1-84, and zoledronic acid on bone microarchitecture and estimated strength in postmenopausal women with osteoporosis: an 18-month open-labeled observational study using HR-pQCT. J Bone Miner Res 28(4):736-745

42. Metcalf LM, Aspray TJ, McCloskey EV (2017) The effects of parathyroid hormone peptides on the peripheral skeleton of postmenopausal women. A systematic review. Bone 99:39-46

43. Chen P, Satterwhite JH, Licata AA, Lewiecki EM, Sipos AA, Misurski DM, Wagman RB (2005) Early changes in biochemical markers of bone formation predict BMD response to teriparatide in postmenopausal women with osteoporosis. J Bone Miner Res 20(6):962-970

44. Drake MT, Srinivasan B, Mödder UI, Peterson JM, McCready LK, Riggs BL, Dwyer D, Stolina M, Kostenuik P, Khosla S (2010) Effects of parathyroid hormone treatment on circulating sclerostin levels in postmenopausal women. J Clin Endocrinol Metab 95(11): 5056-5062

45. Polyzos SA, Anastasilakis AD, Bratengeier C, Woloszczuk W, Papatheodorou A, Terpos E (2012) Serum sclerostin levels positively correlate with lumbar spinal bone mineral density in postmenopausal women - the six-month effect of risedronate and teriparatide. Osteoporos Int 23(3):1171-1176

46. Jeffrey BA et al (2012) Self-reported adherence with the use of a device in a clinical trial as validated by electronic monitors: the VIBES study. BMC Med Res Methodol 12:171

Publisher's note Springer Nature remains neutral with regard to jurisdictional claims in published maps and institutional affiliations. 